Advanced Practice Nurses

\title{
Ein echter Mehrwert
}

Wie kann eine Advanced Practice Nurse ihre Rolle in der Pädiatrie gestalten und welche Hauptkonzepte sind mit der Rolle verknüpft? Unsere Autorin hat während ihres studienbegleitenden Praktikums an einem Hospital in London die Rolle der APN näher kennengelernt und beschreibt anhand eines Fallbeispiels die Arbeit dieser besonderen Pflegefachkräfte.

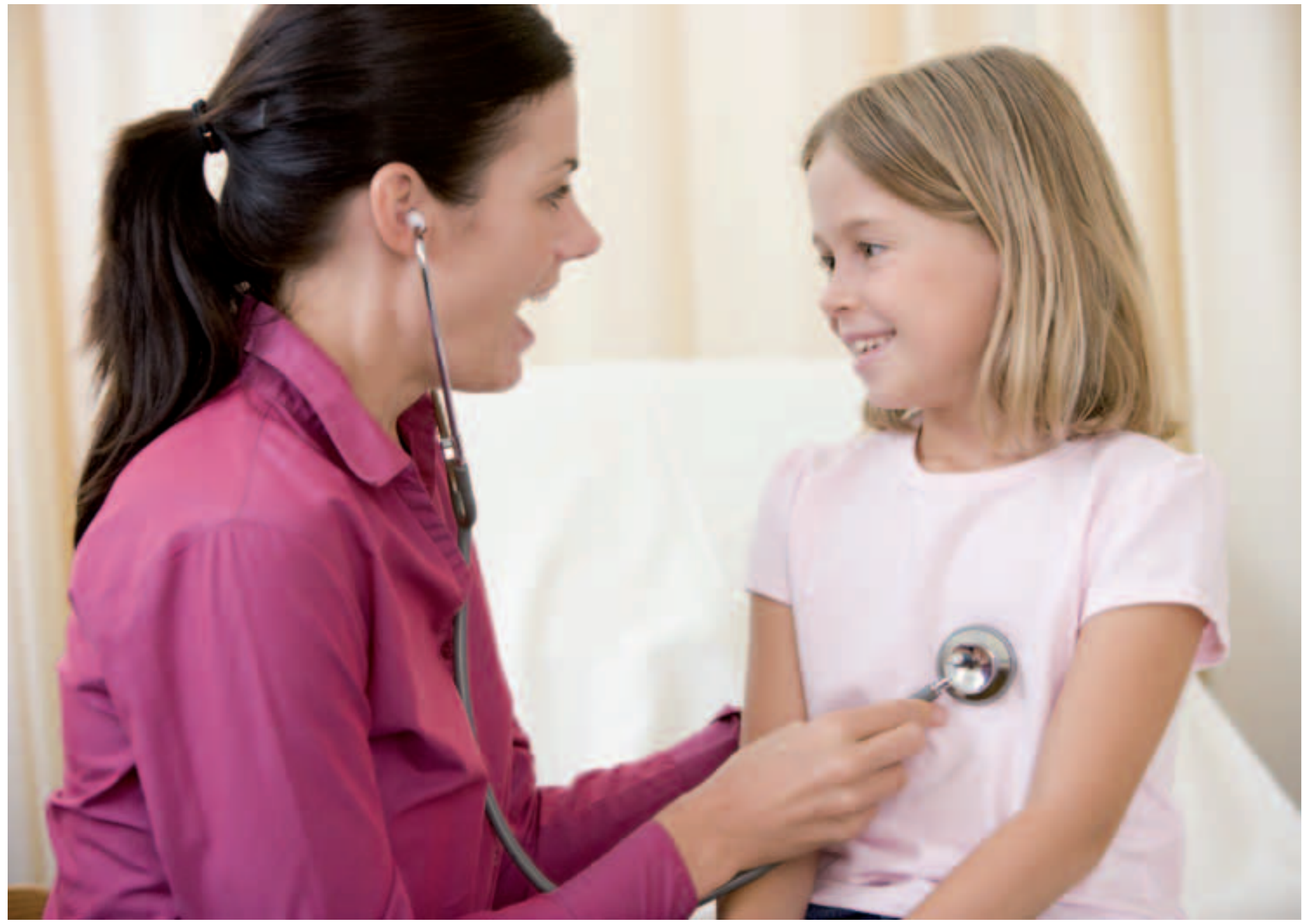

Um mich mit der Praxisrolle der Advanced Practice Nurse (APN) vertieft auseinanderzusetzen, habe ich im Rahmen meines berufsbegleitenden Masterstudiums ein Praktikum am Royal Brompton \& Harefield Hospital in London mit dem APN Pädiatrieteam für Cystische Fibrose (CF) absolviert. Der Einblick in den Pflegealltag einer APN-CF bot mir die Möglichkeit, mich auf meine zukünftige Rolle als APN vorzubereiten, und ermöglichte es mir, die erlernte Theorie während des Studiums in der Praxisumsetzung kennenzulernen. Dabei konnte ich drei APN-Hauptkonzepte - Kontinuität, Ganzheitlichkeit und Caring - als Herzstücke der Arbeit der APN-CF beobachten.
Der allgemeine Wandel im europäischen Gesundheitswesen

Die Zahl der chronisch erkrankten Menschen in Europa steigt unaufhörlich. Zudem ist ein stetiger politischer und personeller Wandel im Gesundheitswesen durch die immer knapperen Ressourcen zu erkennen. Der Druck auf die Gesund- 
heitsversorger, hochstehende Qualität zu leisten und immer höhere Quantitäten zu meistern, erfordert die Entwicklung neuer Versorgungsmodelle. ${ }^{1}$ Seit der Einführung der Diagnosis Related Groups (DRGs) ist eine zunehmende Auslagerung der stationären Versorgung auf die ambulante Betreuung zu beobachten. ${ }^{2}$ Die Gesundheitsversorgung wird dadurch immer mehrdimensionaler und interdisziplinärer. Diese Entwicklung fordert die Koordination der kontinuierlichen Versorgung durch eine Fachperson mit spezifischen und spezialisierten Kompetenzen als Schlüsselperson für verschiedenste Patientengruppen. ${ }^{1}$

\section{Die Rolle Advanced Practice Nurse im Allgemeinen}

„Eine Pflegeexpertin APN ist eine registrierte Pflegefachperson, welche sich durch akademische Ausbildung Expertenwissen, Fähigkeiten zur Entscheidungsfindung bei hoch komplexen Sachverhalten und klinische Kompetenzen für eine erweiterte pflegerische Praxis angeeignet hat. Pflegeexpertinnen APN sind fähig, in unterschiedlichsten Settings vertiefte und erweiterte Rollen zu übernehmen und diese in eigener Verantwortung im interprofessionellen Team auszufüllen. Die Kernkompetenzen einer Pflegeexpertin APN sind: direkte klinische Praxis, Experten-Coaching, Beratung, ethische Entscheidungsfindung, interdisziplinäre Zusammenarbeit, klinisches und fachspezifisches Leadership und Forschungskompetenz". ${ }^{3}$

Die Charakteristik der einzelnen Kernkompetenzen wird vom Kontext und/oder den Bedingungen des jeweiligen Landes geprägt, in dem die APN für die Ausübung ihrer Tätigkeit zugelassen ist. Ein Masterabschluss (Master in Nursing Science) gilt dabei als Voraussetzung. 4

\section{Die Rolle Advanced Practice Nurse in England}

In England gehört die Rolle APN schon länger in die Gesundheitsversorgung. Die Rolle APN wird von der Politik, Gesundheitsfachkreisen sowie von Patienten anerkannt und geschätzt. Somit ist sie aus der Pflegepraxis in England nicht mehr wegzudenken. Auch bei Patienten mit der chronischen Lungenerkrankung Cystische Fibrose (CF) werden APNs eingesetzt.

\section{CF und ihre Bedeutung für die Betroffenen und Familien}

Bei der Cystischen Fibrose handelt es sich um eine angeborene, erblich bedingte Stoffwechselerkrankung, die mit einer Inzidenz von 1:2.500 eine häufige Diagnose bei Neugeborenen ist. ${ }^{5}$ Neuere Studien zeigen, dass $50 \%$ aller CF-Betroffenen weltweit bereits über 18 Jahre alt sind und gute Überlebenschancen bis zum 40. Lebensjahr aufweisen. ${ }^{6}$

Der genetische Defekt der chronisch progredient verlaufenden CF führt zur Elektrolyttransportstörung. Die klinische Manifestation zeigt sich vordergründig durch die obstruktive Lungenerkrankung und exokrine Pankreasinsuffizienz. ${ }^{7}$ Für die Betroffenen beinhaltet die $\mathrm{CF}$ das tägliche Einhalten eines komplexen und aufwendigen Therapiemanagements.8,9 Zur Erhaltung der pulmonalen Funktionen wird täglich mit verschiedenen Medikamenten inhaliert, u.a. Bronchodilatatoren und Antibiotika. ${ }^{8}$ Atemtherapie und Physiotherapie zur Mobilisierung des Lungensekrets müssen täglich absolviert werden. ${ }^{10}$ Die Einnahme diverser Vitamin- und Antibiotikapräparate gehören zum Alltag. ${ }^{8}$ Eine hochkalorische Ernährung und häufig auch die Einnahme von Enzymen sind maßgeblich, um dem krankheitsbedingten Untergewicht entgegenzuwirken und die Gesundheit stabil zu halten. ${ }^{11}$ Bei einer pulmonalen Infektexazerbation wird eine intravenöse Antibiotikatherapie durchgeführt mit dem Ziel, die pulmonale Funktion zu stabilisieren. ${ }^{2}$ Die Infektexazerbationen häufen sich meist mit zunehmendem Alter, wodurch die Antibiotikakuren intensiviert werden und sich Resistenzen bilden können. ${ }^{12}$ Absenzen in der Schule und am Arbeitsplatz steigen an und die Erkrankung kann immer mehr Raum einnehmen. Häufig manifestiert sich zusätzlich ein Diabetes Mellitus. ${ }^{13}$ Aufgrund der Krankheitsprogredienz sehen sich viele CF-Betroffene schlussendlich mit der Option einer Lungentransplantation als ultimative Therapie ihrer fortgeschrittenen Lungenerkrankung konfrontiert. ${ }^{14}$

Folglich sind die Betroffenen ab der Diagnosestellung auf eine kontinuierliche, lebenslange und interdisziplinäre medizinische Versorgung angewiesen. Durch die Zunahme der zu erwartenden Lebensjahre der CF-Betroffenen, der hochkomplexen benötigten medizinischen Versorgung und der sich wandelnden Strukturen in der Betreuung ergibt sich ein Bedarf nach integrierter Versorgung für die CF-Betroffenen. $6,15,16$

\section{Die Rolle APN und die CF-Betroffenen in England}

Im Royal Brompton \& Harefield Hospital, im Pneumologie- und KardiologieZentrum im Stadtteil South Kensington in London, werden circa 320 Kinder mit Cystischer Fibrose und ihre Familien von fünf Pflegefachfrauen mit der Berufsbezeichnung APN-CF betreut. Die APN$\mathrm{CF}$ gehören zu einem interdisziplinären Fachteam, welches die Familien ab der Diagnosestellung, meist kurz nach der Geburt, bis zum 16. Lebensjahr begleitet. Sie sind die primären Ansprechpartner für die Familien, bis der Übertritt in das erwachsene Versorgungssetting stattfindet. Sie führen Hausbesuche bei den Familien durch, machen Konsultationen in der ambulanten CF-Sprechstunde und führen Informations- und Schulungsgespräche mit Betreuungsteams in Krippen, Kindergärten und Schulen. Sie begleiten die Familien während Hospitalisationen und stehen im Alltag per Telefon, E-Mail und Textmitteilung beratend zu Seite. Sie koordinieren die gesamte Behandlung. So verwalten sie beispielsweise auch die bestehende Warteliste für einen stationären Behandlungsplatz.

\section{Praxisumsetzung der Rolle APN-CF in England anhand eines Fallbeispiels} Im Folgenden wird anhand eines konkreten Fallbeispiels die Arbeit der APN-CF illustriert.

\section{Ambulante Betreuung von Anna* vor dem Spitaleintritt durch die APN-CF} Zum Wochenbeginn wurde am Royal Brompton \& Harefield im multidisziplinären Team, bestehend aus dem Pneumologen, der Ernährungsberatung, der Physiotherapie, dem Sozialarbeiter, der Psycho-

\footnotetext{
Name geändert
} 


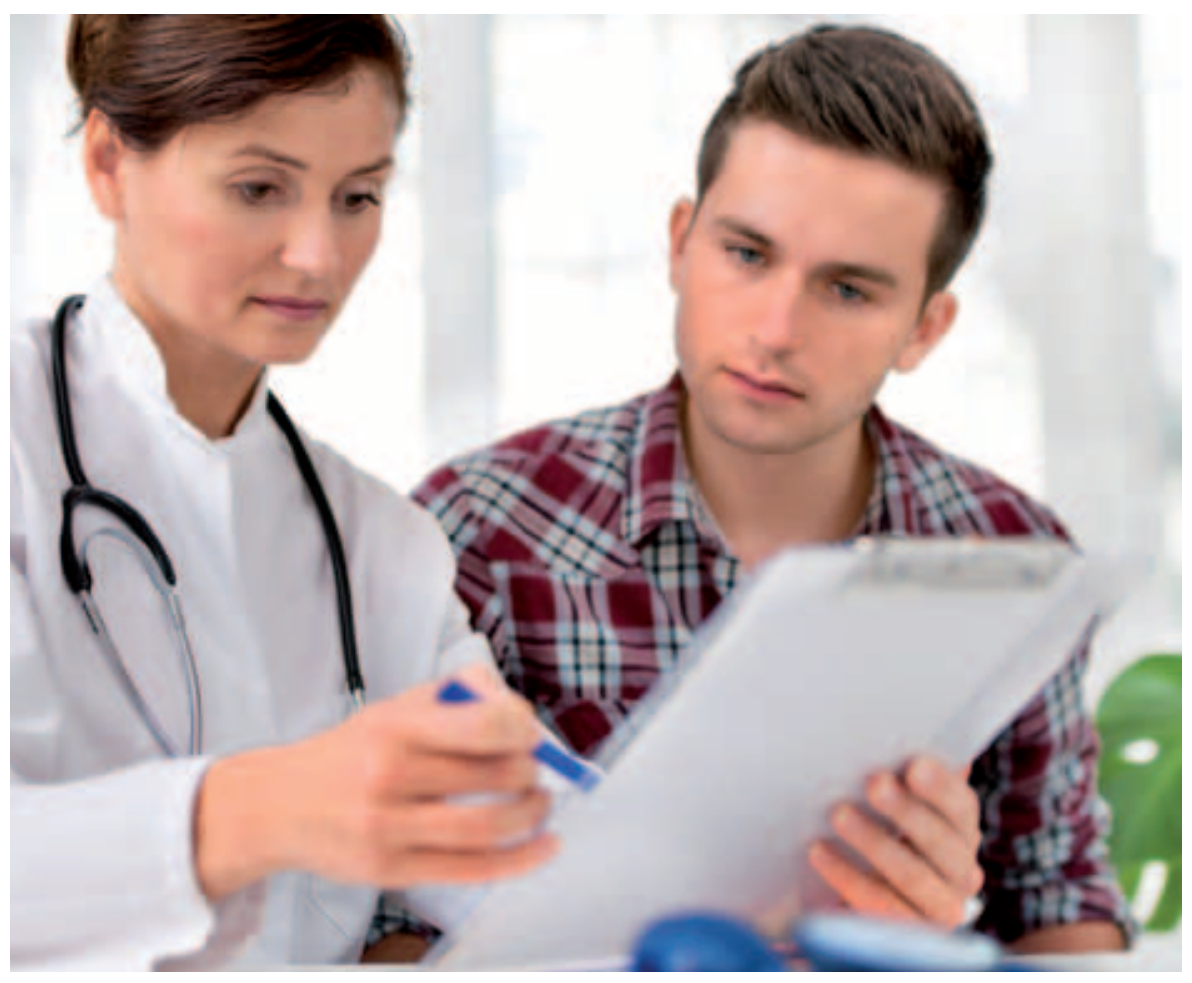

logen und der APN-CF, unter anderem der Fall der 14-jährigen Anna besprochen. Bei Anna stand nach mehreren Wochen Sommerferien der Schulbeginn an. Ihre Mutter hat sich bei der APN-CF telefonisch gemeldet, weil Anna vermehrt hustete, mehr Sekret produzierte und Kurzatmigkeit verspürte. Sie bat um eine Abklärung und schlug eine Hospitalisation für eine intravenöse Antibiotikakur vor. In der Besprechung legten alle Disziplinen ihre Sichtweisen der Situation dar. Das Team einigte sich, dass Anna zu Hause von der APN-CF und der Physiotherapeutin besucht werden sollte, um abzuklären, wie bald die Hospitalisation erfolgen musste. Die APN kontaktierte die Familie telefonisch, um das weitere Vorgehen zu besprechen. Am Telefon passte die APNCF die Inhalationen an und besprach die Medikation.

In den nächsten Tagen wurde Anna zu Hause besucht. Die APN-CF machte ein klinisches Assessment, indem sie Sputum abnahm und die Lungenfunktion testete, um festzustellen, wie sich die Werte seit der letzten Kontrolle vor zwei Monaten verändert hatten. Im Falle von Anna hatten sich die Werte nur geringfügig geändert. Die APN-CF besprach dies mit Anna und ihrer Mutter. Die Inhalationen wurden von der APN-CF erneut angepasst und zusätzlich ein Antibiotikum verordnet. Sie besprachen das Gewicht und die Ernährung. Anna berichtete, dass sie aktuell keine Sondennahrung benötigt, weil ihr Gewicht stabil sei. Die Physiotherapeutin besprach mit Anna ihre Atemphysiotherapie, gab neue Anweisungen und Hilfestellungen zur besseren Kontrolle des akuten Hustenreizes und der Sekretmobilisation. Die Physiotherapeutin und die APN-CF besprachen gemeinsam mit Anna das Behandlungstagebuch und lobten Anna für ihre Fortschritte in der Selbstständigkeit. Die Fragen von Anna und ihrer Mutter wurden umfassend beantwortet.

Anna berichtete, dass sie in einer Woche die Schule wechseln würde und dass sie sich unwohl damit fühle. Sie wisse nicht, wie die Klasse auf sie mit ihrer Krankheit und dem Husten reagieren werde. Die APN-CF vereinbarte mit ihr, die Schule zu kontaktieren, die Lehrperson zu treffen, sie über die Cystische Fibrose aufzuklären und den Umgang damit zu besprechen. Letztlich entschieden die APN-CF und die Physiotherapeutin, dass Anna im Moment keine Notfallaufnahme benötigt, aber zur intravenösen Antibioti- ka- und intensiven Atemphysiotherapie in den nächsten zwei Wochen stationär aufgenommen werden muss. Nach dem Hausbesuch informierte die APN-CF alle involvierten Dienste per E-Mail und platzierte Anna auf der Warteliste zur stationären Aufnahme. In den darauffolgenden Tagen kontaktierte die APN-CF Anna regelmäßig per Telefon und Textmitteilung, um über ihren Zustand informiert zu bleiben und die Therapie bei Bedarf anzupassen.

\section{Die stationäre Betreuung von Anna durch die APN-CF}

Die stationäre Aufnahme von Anna erfolgte kurze Zeit später. Zu diesem Zeitpunkt fand eine interdisziplinäre Besprechung statt, um das stationäre Therapievorgehen festzulegen, über das die Familie bei der gemeinsamen Visite informiert wurde. Während der zweiwöchigen stationären Therapie besuchte die APN-CF Anna regelmäßig und sie organisierte den Austritt, dem eine weitere interdisziplinäre Besprechung voranging, frühzeitig. Die APN-CF unterstützte die Pflegenden auf der Abteilung durch einen regelmäßigen Austausch. Bei Austritt erhielt die Familie Informationen über das neue Therapieprozedere und gab ihre Zustimmung. Alle Disziplinen verfassten einen Austrittsbericht für den behandelnden Hausarzt.

Die ambulante Betreuung von Anna nach dem Spitalaustritt durch die APN-CF

Eine Woche nach dem Spitalaustritt besuchte die APN-CF Anna zu Hause und gemeinsam schauten sie sich alle relevanten Themen an. Die APN-CF testete erneut die Lungenfunktion, um nötigenfalls die Therapie anzupassen. Sie vereinbarten, dass Anna in sechs Wochen die CF-Sprechstunde besuchen und dass sie sich bei Unklarheiten oder Veränderungen jederzeit an die APN-CF wenden soll.

\section{Die APN-Hauptkonzepte: Kontinuität, Ganzheitlichkeit und Caring}

Der Fall von Anna verdeutlicht, dass die APN-CF eine direkte klinische Praxis hat. Sie zeigte Fähigkeiten und Fertigkeiten im Experten-Coaching, in der Beratung, der interdisziplinäre Zusammenarbeit sowie der klinischen und fachspezifischen Führung gegenüber Pflegefachpersonen. 
Dadurch ergeben sich ein umfassendes Betreuungsangebot und eine hohe Betreuungsqualität, die sich durch Kontinuität, Ganzheitlichkeit und Caring auszeichnet. Das APN-CF-Team gewährleistet eine hohe Betreuungskontinuität durch den jahrelangen Kontakt zu den Patienten und ihren Familien. Die Familie von Anna hatte in der APN-CF eine stetige und niederschwellig erreichbare Ansprechpartnerin. Mit der APN-CF können Probleme gebündelt an eine Person adressieren werden, die diese bei Bedarf weitergeleiten kann. Sie koordiniert die Versorgung, wodurch das gesamte Behandlungsvorgehen klar und transparent ist. Die APN-CF nimmt Anna und ihre Familie ganzheitlich wahr, sie können alle Fragen rund um das Thema CF stellen. Nicht nur die medizinische Therapie zählt, sondern die APN-CF bezieht alle Auswirkungen auf das soziale Umfeld mit ein. Dadurch entsteht eine Beziehung getragen von Caring, die zu gegenseitigem Vertrauen und einer ganzheitliche Wahrnehmung von Anna und ihrer Familie führt. Alle Mitglieder der Familie werden als Menschen mit individuellen Bedürfnissen wahrgenommen, denen es bestmöglich gerecht zu werden gilt. Durch diesen Betreuungsansatz geling es trotz eines weitläufigen Einzugsgebiets in London zwischen den Familien und den Gesundheitsversorgern eine umfassende CF-Versorgung anzubieten.

\section{$\rightarrow$ FAZIT}

Obwohl der Zugang zur Gesundheitsversorgung in England und der Schweiz unterschiedlich ist, ist in beiden Systemen der Einsatz einer APN-CF als fachspezifischen Koordinations- und Ansprechperson für alle Beteiligten ein Mehrwert in der Gesundheitsversorgung. Meine persönlichen Erfahrungen in England haben mich von der Praxisrolle APN überzeugt. Der Einsatz einer APN in der Praxis ermöglicht es, Menschen kontinuierlich in ihrer Ganzheitlichkeit wahrzunehmen und sie unter dem Aspekt des Carings zu begleiten. Dies sollte Anreiz sein, für möglichst viele Patientenpopulationen eine solche Betreuung in der Pflegepraxis einzuführen und anzubieten.

\section{$\rightarrow$ LITERATUR}

1 Schweizerische Gesundheitsdirektorenkonferenz E Bundesamt für Gesundheit (BAG). Neue Versorgungsmodelle für die medizinische Grundversorgung. Bern, 2012

2 Swiss ANP. Positionspapier Advanced Nursing Practice in der Schweiz. 2012. Online unter http:// swiss-anp.ch/w/media/Akutelles/Positionspapier_ ANP_Schweiz.pdf, letzter Zugriff 25.01.2015

3 SBK - ASI, IG SwissANP, Schweizerischer Verein für Pflegewissenschaft VFP \& Institut universitaire de formation et de recherche en soins IUFRS. Reglementierung der Pflegeexpertin ANP. 2012. Online unter http://swiss-anp.ch/w/media/Akutelles/Eckpunktepapier.pdf, letzter Zugriff 25.01.2015

4 International Council of Nurses: Definition and characteristics for nurse practitioner/advanced practice nursing roles [official paper]. 2002, June. Online unter www.icn.ch/networks_ ap.htm, letzter Zugriff 25.01.2015 Seite funktioniert nicht!

5 Barben J, Torresani T, Schöni M, Gallati S, Baumgartner M. Neugeborenen-Screening auf zystische Fibrose - bald auch in der Schweiz? Swiss Working Group for Cystic Fibrosis, Schweizerische Gesellschaft für Pädiatrische Pneumologie 2008, 19: 22-23

6 Tuchman L, Schwartz L, Sawicki G, Britto M. Cystic fibrosis and transition to adult medical care. Pediatrics 2010, 125: 566-573

7 Lindemann H, Dockter G, Tümmler B. Mukoviszidose. Zystische Fibrose. Stuttgart: Thieme; 2004

8 Mogayzel PJ Jr, Naureckas ET, Robinson KA, Mueller G, Hadjiliadis D, Hoag JB, Lubsch L et al. Cystic fibrosis pulmonary guidelines. Chronic medications for maintenance of lung health. American journal of respiratory and critical care medicine 2013, 187(7): 680-689

9 Van Staa A, van der Stege AH, Jedeloo S, Moll HA, Hilberink SR. Readiness to Transfer to Adult Care of Adolescents with Chronic Conditions: Exploration of Associated Factors. Journal of Adolescent Health 2010, 48(3): 295-302

10 Holland AE, Button BM. Physiotherapy for cystic fibrosis in Australia: knowledge and acceptance of the Consensus Statement recommendations. Respirology 2013, 18(4): 652-656

11 Haupt ME, Kwasny MJ, Schechter MS, McColley SA. Pancreatic enzyme replacement therapy dosing and nutritional outcomes in children with cystic fibrosis. The Journal of paediatrics 2014, 164(5): 1110-1115.e1

12 Goss CH, Burns JL. Exacerbations in cystic fibrosis 1: Epidemiology and pathogenesis. Thorax 2007, 62(4): 360-367

13 Ode KL, Moran A. New insights into cystic fibrosis-related diabetes in children. The Lancet. Diabetes $\mathcal{E}$ Endocrinology 2013, 1(1): 52-58

14 Hirche TO, Knoop C, Hebestreit H, Shimmin D, Solé A, Elborn JS, Ellemunter H et al. Practical Guidelines: Lung Transplantation in Patients with Cystic Fibrosis. Pulmonary medicine 2014, DOI: $10.1155 / 2014 / 621342$
15 Beacham BL, Deatrick JA. Health care autonomy in children with chronic conditions: implications for self-care and family management. The Nursing clinics of North America 2013, 48(2): 305-317

16 Dyer J. Cystic fibrosis nurse specialist: a key role. Journal of the Royal Society of Medicine 1997, 21-5

\section{G AUTORINNEN}

Francesca Hauser-Piatti, Dipl. Pflegefachfrau, BScN, cand. MScN

Diplomierte Pflegefachfrau in der Pädiatrie, seit sechs Jahren auf einer gemischten medizini-

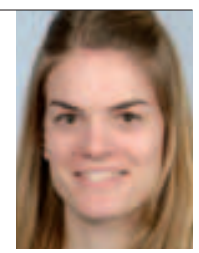
schen Kinderabteilung mit Patienten im Alter von zwei Wochen bis 16 Jahre beschäftigt. Ihr spezielles Interesse gilt den Familien mit CF-Kindern. Thema Ihrer Bachelorarbeit: „Needs in Transition: Was für Bedürfnisse haben CF-Betroffene und ihre Familien während der Transition von der Pädiatrie zum Erwachsenenversorgungssetting?". Aufbauend auf diesen Erkenntnissen führt sie als Masterarbeit eine retrospektive, qualitative Erhebung des Erlebens von CF-Betroffenen und ihren Angehörigen während der Transition zwischen einem pädiatrischen und einem Erwachsenenversorgungssetting und eine Evaluation des interdisziplinären Transitionsprogramms durch.

E-Mail: piattfra@students.zhaw.ch

\section{Dr. rer. medic Daniela}

Händler-Schuster

Dozentin des Instituts für Pflege an der Zürcher Hochschule für Angewandte Wissenschaften in Winterthur (ZHAW). Sie leitet verschiedene Module im Studiengang Master of Science in Pflege und ist Projektleiterin im Bereich Forschung und Entwicklung Pflege.

E-Mail: haed@zhaw.ch

Prof. Dr. Romy Mahrer-Imhof Professorin für familienzentrierte Pflege an der Zürcher Hochschule für Angewandte Wissenschaften in Winterthur (ZHAW). Sie leitet den Studiengang Master of Science in Pflege. Sie setzt sich seit Jahren für APN ein und unterrichtet zu Rollenentwicklung der Advanced Practice Nurses in verschiedenen Studiengängen. E-Mail: mahr@zhaw.ch

\section{BIBLIOGRAFIE}

DOI $x x x$

JuKiP 2015; 4: 72-75

(C) Georg Thieme Verlag KG

Stuttgart · New York · ISSN 1439-2569 